\title{
Spontaneous Pneumoperitoneum: Intraperitoneal Air without Hollow Viscus Injury
}

\author{
Dong Hun Kim, Jeongseok Yun \\ Department of Surgery, Trauma Center, Dankook University Hospital, Cheonan, Korea
}

Spontaneous pneumoperitoneum can be confusing in diagnosis of hollow viscus injury. In this report, we describe a blunt trauma patient with extraluminal intraperitoneal air in the absence of bowel perforation without a chest injury. Abdominal physical examination revealed peritoneal irritation sign. Abdominal computed tomography showed intraperitoneal air along the right perihepatic area and a right retroperitoneal hematoma. Intraoperatively, we observed a right adrenal hematoma and serosal tearing of the gallbladder and ascending colon without bowel perforation.

(Trauma Image Proced 2016(1):21-22)

Key Words: Pneumoperitoneum; Abdominal Cavity; Intestines

\section{CASE}

A 45-year-old man with cerebral palsy was involved in a traffic accident and sustained a blunt abdominal injury. On arrival, he was alert with the following vital signs: blood pressure, 118/72 $\mathrm{mmHg}$; pulse, 80/min; respiratory rate, $16 / \mathrm{min}$; and body temperature, $38.0^{\circ} \mathrm{C}$. Physical examination of the abdomen revealed tenderness and rebound tenderness in the right upper quadrant, and diminished bowel sounds. Supine chest radiography did not show any specific findings. Abdomen computed tomography (CT) revealed a right retroperitoneal hematoma involving the right adrenal gland and perirenal space and a scanty amount of intraperitoneal air along the right perihepatic area (Fig. 1.). Exploration of the abdominal cavity revealed a right adrenal hematoma, perirenal hematoma, and serosal tearing of the gallbladder and ascending colon. There was no gastrointestinal tract perforation. The patient resumed oral intake 3 days after cholecystectomy and right adrenalectomy without any complications.

\section{DISCUSSION}

Spontaneous pneumoperitoneum, described as the presence of air confined to the inner layer of the abdominal wall and external to the parietal peritoneum, may masquerade as true pneumoperitoneum (1), thereby representing a diagnostic pitfall. It should be recognized that extraluminal intra- or retroperitoneal air rarely occurs without gastrointestinal tract perforation. It is associated with various causes, such as mechanical ventilation and pulmonary barotrauma, peritoneal lavage before computed tomography (CT), pneumothorax, chest injury, entry of air via the female genital tract, and intraperitoneal laceration of the bladder secondary to cysto-

Received: July 18, 2016 Revised: August 2, 2016 Accepted: August 17, 2016

Correspondence to: Dong Hun Kim, Department of Surgery, Trauma Center, Dankook University Hospital, 201 Manghyang-ro, Dongnam-gu, Cheonan, Chungnam 330-715, Korea

Tel: 82-41-550-7119, Fax: 82-41-550-0039, E-mail: saint7331@gmail.com

Copyright (c) 2016 Korean Association for Research, Procedures and Education on Trauma. All rights reserved.

(c) This is an open-access article distributed under the terms of the Creative Commons Attribution Non-Commercial License (http://creativecommons.org/ licenses/by-nc/4.0) which permits unrestricted noncommercial use, distribution, and reproduction in any medium, provided the original work is properly cited 

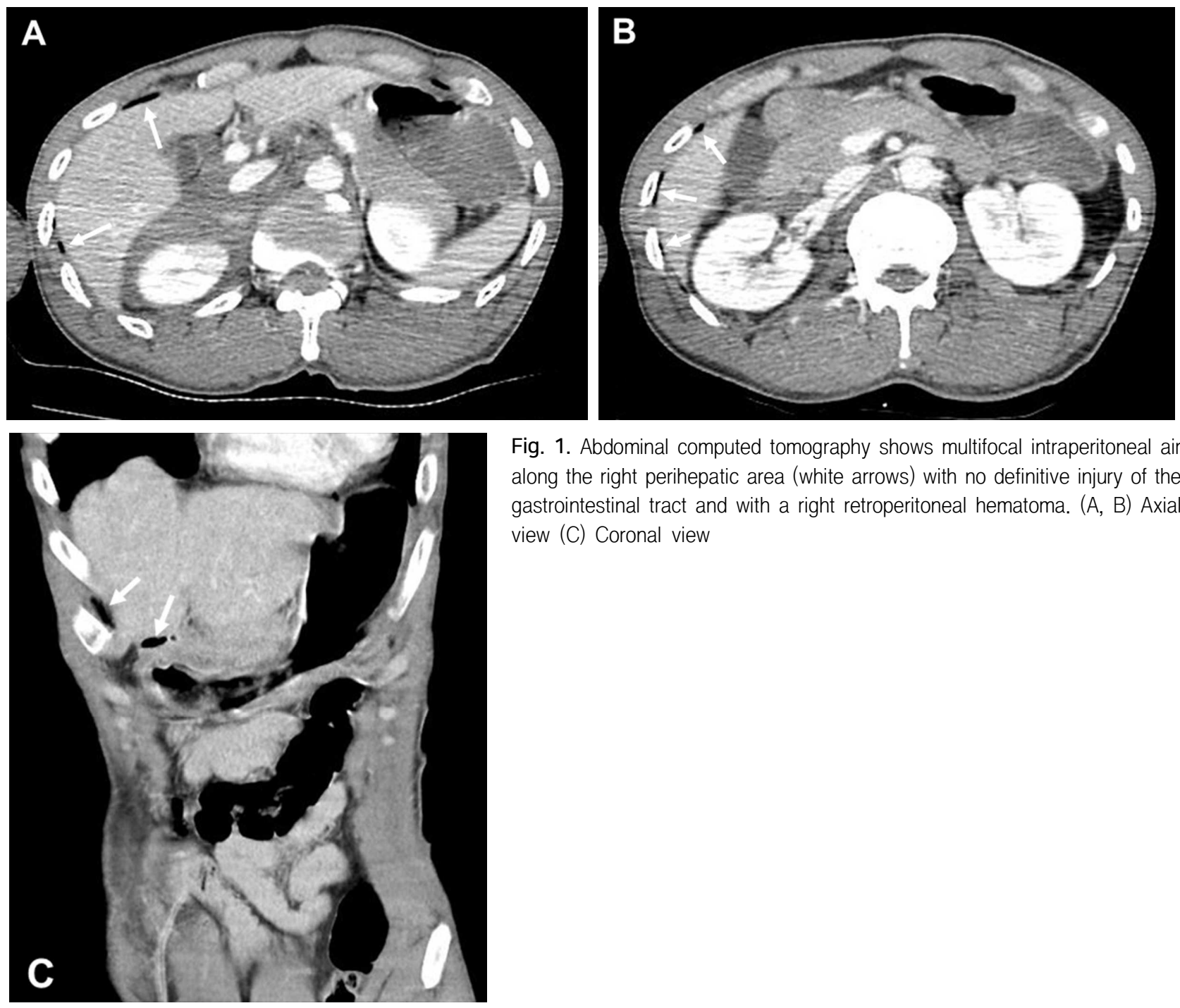

Fig. 1. Abdominal computed tomography shows multifocal intraperitoneal air along the right perihepatic area (white arrows) with no definitive injury of the gastrointestinal tract and with a right retroperitoneal hematoma. (A, B) Axial view (C) Coronal view

graphy $(1,2)$. A repeat CT examination within 6-8 h may help determine the significance of less specific signs of bowel and mesenteric injuries, such as focal bowel wall thickening, mesenteric fat stranding with focal fluid and hematoma, and intraperitoneal or retroperitoneal fluid (3). Thus, reevaluation with additional CT findings that are indicative of gastrointestinal tract perforation may strengthen the significance of the extraluminal free air.

\section{CONFLICT OF INTEREST}

No potential conflict of interest relevant to this article was reported.

\section{REFERENCES}

1. Brody JM, Leighton DB, Murphy BL, Abbott GF, Vaccaro JP, Jagminas L, et al. CT of blunt trauma bowel and mesenteric injury: typical findings and pitfalls in diagnosis. Radiographics : a review publication of the Radiological Society of North America, Inc. 2000;20(6):1525-36; discussion 36-7.

2. Hamilton P, Rizoli S, McLellan B, Murphy J. Significance of intra-abdominal extraluminal air detected by CT scan in blunt abdominal trauma. The Journal of trauma. 1995; 39(2):331-3.

3. Brofman N, Atri M, Hanson JM, Grinblat L, Chughtai T, Brenneman F. Evaluation of bowel and mesenteric blunt trauma with multidetector CT. Radiographics : a review publication of the Radiological Society of North America, Inc. 2006;26(4):1119-31. 